\title{
Modelado Físico como Estrategia para el Diseño de los Atributos de un Nuevo Producto
}

\author{
Juan C. Briede-Westermeyer ${ }^{(1)}$, Isabel M. Leal-Figueroa ${ }^{(1)}$ y Cristhian E. Pérez-Villalobos ${ }^{(2)}$ \\ (1) Universidad del Bío-Bío, Departamento de Arte y Tecnologías del Diseño, Avda. Collao 1202, \\ Casilla 5-C, Concepción-Chile (e-mail: jbriede@ubiobio.cl; ileal@ubiobio.cl). \\ (2) Universidad de Concepción, Departamento de Educación Médica, Víctor Lamas 1290, Concepción- \\ Chile (e-mail: cperezv@udec.cl).
}

Recibido Abr. 12, 2017; Aceptado Jun. 7, 2017; Versión final Ago. 18, 2017, Publicado Dic. 2017

\begin{abstract}
Resumen
En el presente estudio exploratorio se describe y analiza una experiencia docente en la que se abordó diseño conceptual utilizando el enfoque sistémico de Hernandis y Briede del año 2009, pero abordando la integración de los atributos teóricos del producto en una maqueta física del espacio del diseño. Esta estrategia busca facilitar la gestión, y transferencia de los atributos teóricos que se integraron en el espacio del diseño a la propuesta de diseño final. En el estudio, se aplicó una encuesta de percepción a los alumnos del taller de diseño centrado en el usuario el año 2013, ofrecido por Departamento de Arte y Tecnologías del Diseño de la Universidad del Bío-Bío, en Chile. Los resultados indican que el proceso constructivo permitió a los alumnos conectar lo teorizado con su aplicación en el diseño final. El modelado físico, como soporte didáctico, facilitó el proceso de análisis y la toma de decisiones relacionado con la aplicación de los conceptos teóricos estudiados.
\end{abstract}

Palabras clave: diseño conceptual; modelado conceptual; maqueta; espacio de diseño; educación en diseño

\section{Physical Modeling as an Attribute Design Strategy of a New Product}

\begin{abstract}
In this exploratory study a teaching experience in which conceptual design was addressed using the systemic approach by Hernandis and Briede of 2009, but covering the integration of the theoretical attributes of the product in a physical model of the design's space is described and analyzed. This strategy aims at facilitating the management and transfer of the theoretical attributes that are integrated in the design's space for the final design proposal. In the study, a perception survey was applied to students of the user-centered design workshop of the year 2013, offered by the Dept. of Art and Design Technologies of the University of Bío-Bío, in Chile. The results indicate that the construction process allowed students to connect theory with their application in the final design. The physical modeling, as didactic support, facilitated the analysis and decision-making process related to the application of the theoretical concepts already studied.
\end{abstract}

Keywords: conceptual design; concurrent modelling; model; design space; design education 


\section{INTRODUCCIÓN}

El diseño y desarrollo de nuevos productos es una actividad clave abordada desde hace décadas por las empresas para ser competitivas en los mercados, buscando satisfacer las necesidades de las personas. Un proceso de diseño y desarrollo, para que esa búsqueda sea exitosa, debe responder y considerar un sinnúmero de variables y factores, tanto de función, usabilidad, apariencia y mercado, solo por nombrar algunas, lo que se demuestra en las dimensiones de la complejidad del producto (Aguayo, Soltero, 2003; Basu et al.,2013).

Esta actividad se aborda generalmente a través de la definición de las necesidades de los usuarios y el establecimiento de las especificaciones del nicho (Ulrich, Eppinger, 2011) donde se estudian los mercados se definen requerimientos para pasar a una fase de generación de conceptos; en la cual se definen conceptos y los atributos teóricos que debería contemplar el producto. Esta definición teórica será la base para poder generar la propuesta formal del producto; con sus características formales, tamaño, proporciones considerando la o las funciones y la accesibilidad por parte del usuario para poder usufructuar de su uso. Y responda en síntesis a las especificaciones y necesidades de la etapa anterior. Dentro de los enfoques tradicionales para abordar el diseño de productos: los provenientes de la ingeniería que se distingue por su secuencialidad prescriptiva (Asimow, 1962; Hubka y Eder, 1996), donde se busca sistematizar el proceso de diseño mediante operaciones lógicas que van de acuerdo con las necesidades del diseño con el objetivo de aumentar la probabilidad de alcanzar el éxito técnico y económico (Pahl y Beitz, 1996). Así como enfoques de gran popularidad y aceptación como los de Ulrich y Eppinger (2011) que continuamente se actualiza con nuevas versiones. Todos ellos comparten una etapa de generación del concepto y su posterior representación en forma de producto, vínculo que se gestiona tradicionalmente a través de una lista de atributos y especificaciones para ser utilizado como base para el diseño conceptual. Esta fase de síntesis es muy importante, pero al ser trabajada en una dimensión abstracta, sin referentes tangibles o concretos, se dificulta su definición y relación con la propuesta formal y corpórea del producto.

El diseño sistémico por su parte, se refiere a sistemas de orden superior que implican múltiples subsistemas (Jones y Kilma, 2018). Al integrar el pensamiento sistémico y sus métodos, el diseño sistémico lleva el diseño centrado en el humano hacia servicios complejos y con múltiples partes interesadas (Jones y Kijima, 2018). A medida que los diseñadores se involucran en áreas problemáticas cada vez más complejas, ya no es suficiente basarse en creatividad individual y los métodos contemporáneos para pensar en diseño (Ryan, 2014). El diseño sistémico pretende ayudar a los equipos para ver un desafío complejo de una manera diferente, y a traducir esta "nueva visión" en nuevas intervenciones. (Ryan, 2014) El diseño sistémico permite a diversos equipos desarrollar una perspectiva elevada del desafío y traducir los nuevos conocimientos en acción rápida a través de un ciclo iterativo de encuadre, formulación, generación, reflexión, consulta y facilitación. Por su parte Jones, (2014) identificó un conjunto de principios sistémicos compartidos entre la práctica de diseño y la teoría de sistemas, que podrían guiar el pensamiento de diseño y tal vez evaluar el razonamiento sistémico de las propuestas de diseño. Estos principios de diseño relevantes exigen el descubrimiento de relaciones metodológicas entre la teoría de sistemas y el diseño.

Para facilitar esta brecha entre concepto y forma existen enfoques, dentro de los cuales se destaca el propuesto por Hernandis e Iribarren (1999), el que se distingue porque abordan el análisis y modelado del producto, seleccionando las variables que vienen del sistema exterior (contexto) y articulándolas dentro del sistema de referencia que es el producto en cuestión. Esto permite analizar sus múltiples relaciones, causa, efecto, etc. Para determinar cuál es la combinación adecuada para responder a los requerimientos y necesidades planteadas. Pero su relevancia radica en que considera que el sistema de referencia, a su vez, se desagrega en subsistemas fundamentales de forma, función y ergonomía (fo, fu, er), cada uno de los cuales se relaciona sinérgicamente con los otros, generando una interdependencia dinámica. Así, aplicaciones del enfoque sistémico para el diseño conceptual de productos, implican gestionar una complejidad y factores claves en entornos, donde la experiencia es fundamental (Briede et al., 2016).

Así, el enfoque propuesto por Hernandis (2003) busca desagregar sistémicamente la llamada lista de atributos a través de subsistemas fundamentales de forma, función y ergonomía, a los cuales se les asignan los atributos descritos a nivel conceptual y teórico que deberá considerar el diseñador. A partir de esto, es posible transitar a una fase de modelización geométrica, donde, precisamente se asocian estos atributos a geometrías con el objeto de mapear en un espacio tridimensional dichas variables. Este proceso paso a paso permite supervisar el traspaso, transformación o modificación de la forma en que los atributos seleccionados responden a los requerimientos y normativas del producto. Su aplicación en investigación, postgrado y desarrollo de productos ha reportado ventajas claras a diseñadores experimentados, ya que la mayor dificultad dentro del proceso de diseño radica en el alto grado de abstracción de las variables (Hernandis, 2016). Estos mismos modelos han sido utilizados de base para la enseñanza del diseño. Sin embargo, Corremans y Mulder-Nijkamp (2016) constatan que los diseñadores jóvenes con poca experiencia requieren de apoyos para 
encarar procesos de bloqueos al momento de enfrentarse a una página en blanco. Estos apoyos también deben ser extensivos a otras etapas del proceso de orientación sistemática, como es el modelado conceptual. Hernandis y Briede (2009) presentan una propuesta educativa para abordar el diseño conceptual utilizando modelos conceptuales. Esta propuesta se basa en el planteamiento sistémico de Hernandis, 2003 para definir una serie de etapas para abordar el diseño conceptual de un producto: Definición del Producto, Estudio de la Competencia, Definición de Modelos Conceptuales (forma, función y ergonomía), Espacio del Diseño y Diseño Conceptual. Este método fue aplicado en alumnos de tercer ciclo en la Universidad Politécnica de Valencia en el curso de Ocio y Automoción. Si bien el proceso permitió guiar la toma de decisiones, persistía un alto grado de subjetividad y ambigüedad entre la fase de modelado geométrico y las propuestas formales de conceptos. Es decir, entre el espacio del diseño y las propuestas de productos.

En este sentido, el método propuesto busca acercar el espacio del diseño al proceso de desarrollo formal, y confrontarlo con la dimensión concreta entre la representación abstracta del concepto/atributo y su materialización concreta. Y contribuir a que el estudiante promueva el proceso dialéctico de exploración. Por ejemplo, uno define una superficie protectora. Esto puede interpretarse de múltiples modos en el producto: Una rejilla rígida. Una membrana elástica. Elementos separadores que por cercanía y modularidad generen una suerte de superficie o manto, etc. Es promover la interpretación y mostrar que la "maqueta del espacio del diseño" no es la "maqueta del producto". En el estudio realizado por Gudur (2016) asociado en los desafíos en la enseñanza de habilidades para pensar y reflexionar en diseño a estudiantes novicios. Donde la edad y la experticia del estudiante marca la diferencia. En su estudio reportaron que algunos estudiantes novatos experimentaron dificultades en el trato con el pensamiento abstracto en comparación con los estudiantes más experimentados. En una investigación respecto de los medios y representaciones en la educación de diseño del producto (Babapour et al., 2014) se advierte como dentro del rol de las actividades de externalización aparece el modelado físico (maqueta) como un medio que contribuye dentro de los roles físicos; corporeizar ideas, transformaciones radicales como incrementales, roles sensorios como dimensión y proporción, diferencia de ángulos y perspectivas (ayudas visuales), además de poder asociarlo con la funcionalidad y usabilidad (ayudas táctiles). Experiencias relacionadas con la formación en diseño sistémico en estudiantes de postgrado: master. Donde se integra el diseño sistémico y el eco-diseño, enseñando una metodología de tres etapas para ser aplicada en el proyecto de diseño sistémico: Investigación de escritorio, estudio de campo y síntesis de investigación apoyándose en el visualización del diseño de la información a través de un "mapa visual" que permitió organizar el diagnóstico efectuado, considerando e integrando todas las variables estudiadas (Silva, 2016)

\section{ENFOQUE DE DISEÑO}

El diseño centrado en el usuario (DCU) ubica al ser humano como centro, inicio y fin del proceso de diseño (Krippendorf, 2006). En contextos específicos, como el diseño de las aplicaciones web, considera al usuario como un sujeto clave al momento de evaluar, sugerir y hasta diseñar parte de las aplicaciones (Chauncey, 2010). Si lo llevamos al diseño de productos existen variados métodos para registrar, sistematizar y utilizar la voz del usuario (Tu et al., 2011). El presente trabajo documenta parte de la experiencia, específicamente el diagnóstico, en el Taller Diseño Centrado en el Usuario [DCU], parte del proceso formativo de la carrera de Diseño Industrial de la Universidad del Bío-Bío, cuyo enfoque metodológico busca responder a las características específicas del contexto y asumir el rol social del diseño, explorando posibles áreas de desempeño a partir del estudio de comunidades vulnerables (Briede et al., 2011). La metodología DCU tiene como principio diseñar un producto basándose en requerimientos y necesidades de un usuario real en su contexto igualmente real (Lim et al., 2012). Para ello, se implementa un enfoque participativo (Wilkinson, 2014) que busca integrar activamente a los actores durante el proceso de diseño, cambiando del paradigma de diseñar "para la gente" a diseñar "con la gente". La gestión y consideración tanto de requerimientos, limitaciones, así como la definición de atributos y principios que puedan responder a las necesidades del usuario hacen necesario utilizar métodos de diseño que permitan registrar y gestionar dicha información durante la fase de diseño conceptual. Se utilizó el enfoque sistémico, con el propósito de apoyar la gestion la complejidad de las variables y atributos del producto y estimular la reflexión y análisis para apoyar el proceso de toma de decisiones. Descomponiendo los atributos del potencial producto en los tres subsistemas ya mencionados, es que permite y facilita la asociación posterior del diseño a tres tipologías de representación geométrica: Volúmenes, Superficies y Límites de Contorno. La finalidad de esto es exponer tales representaciones en una maqueta tridimensional que facilita identificar jerarquías, develar sus valores (atributos), relaciones, posibles consecuencias y efectos de la propuesta por diseñar. Se busca abordar una temática altamente relevante para la comunidad vulnerable, con situación de dependencia que pone en riesgo la autodeterminación su libre elección en sus ideales de vida y desarrollo (Correa, 2011) y que es solicitada por sus miembros, lo que implica que la propuesta de diseño debe atender a la problemática social presente que emerge, con una mirada contextualizadora que considere el contexto regional y nacional, abordando la estrategia y enfoque del diseño inclusivo (Coleman et al., 2007), con el fin de considerar y ser capaz de trabajar con contextos en riesgo social de nuestro país. 
El presente trabajo documenta parte de la experiencia desarrollada en el Taller Diseño Centrado en el Usuario [DCU], como parte del proceso formativo de la carrera de Diseño Industrial de la Universidad del Bío-Bío, cuyo enfoque metodológico busca responder a las necesidades y características específicas del contexto y asumir el rol social del diseño, explorando posibles áreas de desempeño a partir del estudio de comunidades vulnerables (Briede et al., 2011). Es así como el foco del taller se centró en el "emprendimiento callejero", como temática y contexto de estudio para detectar problemáticas y/u oportunidades para desarrollar productos. Debido a que los llamados vendedores ambulantes son un fenómeno en aumento (Rodríguez, 2015), sujetos que poseen permiso legal esporádico o excepcional y que buscan acceder a un ingreso vendiendo una diversidad de productos manufacturados, tales como artesanías, alimentos, joyas y otros. Estos son parte del grupo de trabajadores que pertenecen a la economía informal, que se caracteriza por carecer de contrato, dedicarse a actividades de subsistencia y carecer de protecciones jurídicas y reglamentarias, lo que los convierte en trabajadores de alta vulnerabilidad (Moyano, Castillo y Lizana, 2008). El objetivo principal de este taller fue aplicar la metodología de diseño centrada en el usuario para diseñar un producto que respondiera a las necesidades de este grupo de trabajadores, contando como objetivos específicos: 1) Comprender y aplicar métodos DCU en el diseño de un producto, 2) Integrar al usuario en el desarrollo del proyecto tanto en las de definición del problema como en el proceso de diseño como proceso de ideación, selección y co-creación, y 3) Comprender, valorar y vivenciar el rol social del diseño ejerciéndolo con responsabilidad social.

\section{MATERIALES Y MÉTODOS}

El presente trabajo documenta los resultados de la aplicación del enfoque sistémico en el Taller de Diseño Centrado en el Usuario [DCU] durante la etapa de modelado conceptual. La investigación documenta su implementación durante la etapa de diseño conceptual del taller [DCU] (Briede et al., 2014). En éste se consideró el desarrollo de modelos conceptuales para el análisis desagregado del producto en los subsistemas fundamentales de forma, función y ergonomía (Hernandis, Briede, 2009). En la tabla 1 se puede observar el momento, dentro de la secuencia de actividades del taller, en que se aplicó la metodología que derivó en el diseño de un nuevo producto. Donde las etapas demuestran la manera en que se van definiendo aspectos claves como: la identificación del problema y necesidad, mapear el contexto de uso, definir limitaciones y posibles restricciones, jerarquización de los problemas para que el propio usuario seleccione aquella más relevante. Esto se concreta finalmente en la definición de objetivos del producto y el desarrollo de la propuesta conceptual y formal a través de un proceso de exploración continua, donde la propuesta se evalúa según su aporte a través de criterios de diseño que el propio equipo define.

Tabla 1: Secuencia de etapas abordadas por los alumnos del taller para diseñar un producto siguiendo el enfoque DCU

\begin{tabular}{|l|l|l|}
\hline \multicolumn{1}{|c|}{ Etapa } & \multicolumn{1}{|c|}{ Métodos y Herramientas } & \multicolumn{1}{c|}{ Objetivos } \\
\hline Inmersión & Observación & $\begin{array}{l}\text { Conocer y familiarizarse con el } \\
\text { contexto de estudio }\end{array}$ \\
\hline Invitación & Entrevista informal & Establecer contacto con el usuario. \\
\hline Observación del Contexto & $\begin{array}{l}\text { Observación /Perfil de Usuario. Secuencia } \\
\text { Diaria }\end{array}$ & $\begin{array}{l}\text { Aplicar enfoques de investigación en } \\
\text { diseño para conocer al usuario y las } \\
\text { actividades que realiza en su rutina } \\
\text { diaria. }\end{array}$ \\
\hline Enfocar Problema & Clasificación de tarjetas, Jerarquización & $\begin{array}{l}\text { Valorizar y seleccionar el problema } \\
\text { más relevante. }\end{array}$ \\
\hline Diseño Conceptual & $\begin{array}{l}\text { Propuesta Conceptual; Metodología } \\
\text { Sistémica }\end{array}$ & $\begin{array}{l}\text { Desarrollar una propuesta a nivel } \\
\text { conceptual. }\end{array}$ \\
\hline Validación y Testeo & Prototipo , Lista de verificación, Entrevistas & $\begin{array}{l}\text { Validar y testear el prototipo real del } \\
\text { producto diseñado. }\end{array}$ \\
\hline
\end{tabular}

Productos esperados de la actividad

El taller [DCU] del año 2013 abordó la problemática del micro emprendimiento callejero como foco de estudio. En dicha ocasión el taller se dividió en grupos formados por 3 o 4 estudiantes, con el fin de poder abordar las etapas del Proyecto en detalle. Para ilustrar la aplicación de las fases claves del enfoque propuesto se utilizará el proyecto desarrollado por el grupo de estudiantes (Dafne Jara, Sandra Herrera y Lenin Palma). Éste abordaba la definición de los aspectos claves de todo proyecto de diseño utilizando la metodología abordada por la Escuela de Diseño de la Universidad del Bío-Bío. Estos aspectos se definen en la tabla 2. 
Tabla 2: Aspectos teóricos troncales dentro del diseño conceptual del producto

\begin{tabular}{|l|l|}
\hline Tema & Implementación objetual para apoyo a micro-emprendimientos \\
\hline Caso & Exhibición de ponchos sobre paredes de material ligero \\
\hline $\begin{array}{l}\text { Propuesta } \\
\text { Conceptual }\end{array}$ & $\begin{array}{l}\text { Exhibidor de sobreposición radial para el almacenaje colgado de ponchos en tiendas de } \\
\text { material ligero }\end{array}$ \\
\hline Objetivo General & $\begin{array}{l}\text { Mejorar la visualización en la exhibición de ponchos en tiendas de material ligero, con el fin } \\
\text { de disminuir su manipulación en el acto de venta. }\end{array}$ \\
\hline Objetivos Específicos & $\begin{array}{l}\text { i) Exponer generando un orden en el almacenamiento de ponchos, para facilitar alcance y } \\
\text { reducir los tiempos de búsqueda del producto. } \\
\text { ii) Mejorar la visualización de cada poncho, considerando sus principales atributos para } \\
\text { persuadir destacando sus virtudes en primera instancia. } \\
\text { iii) Lograr que la exhibición de los ponchos sea completa, de modo que salga del exhibidor } \\
\text { sólo al momento de prueba. }\end{array}$ \\
\hline
\end{tabular}

La realización de la etapa de diseño conceptual considerando el enfoque sistémico, implicaba la realización de los siguientes productos: análisis de diseños pre-existentes, ajuste de propuesta conceptual (opcional) y definición de modelos conceptuales.

Análisis de diseños pre-existentes: se busca realizar un estudio de mercado respecto de los productos y tipologías de productos que comparten las mismas propiedades y funciones. Es decir, la competencia existente en el mercado para el producto utilizando para ello una matriz como la que se puede observar en la figura 1 que considera evaluar los aspectos atingentes a la forma, función y ergonomía pero de forma desagregada, considerando además por cada uno de ellos las ventajas y desventajas que pueden presentar.

\begin{tabular}{|c|c|c|c|c|c|c|}
\hline PRODUCTO & \multicolumn{2}{|c|}{ F OR M A } & \multicolumn{2}{|c|}{ FUNCIÓN } & \multicolumn{2}{|c|}{ ERGONOMÍA } \\
\hline & VENTAJAS & DESVENTAJAS & VENTAJAS & DESVENTAJAS & VENTAJAS & DESVENTAJAS \\
\hline & $\begin{array}{l}\text { Al ser abierto resulta } \\
\text { fácil de utilizar; } y \text { su } \\
\text { alturar permite } \\
\text { abarcar } \quad \text { mayor } \\
\text { espacio. }\end{array}$ & $\begin{array}{l}\text { Las bandejas al estar } \\
\text { muy juntas una con } \\
\text { la otra no permite el } \\
\text { amontonamiento de } \\
\text { ropa. }\end{array}$ & $\begin{array}{l}\text { Al contar con rieles, } \\
\text { hace que la bandeja } \\
\text { se desliza rápido y } \\
\text { de una manera } \\
\text { fluida. }\end{array}$ & $\begin{array}{l}\text { Poca capacidad para } \\
\text { almacenamiento de } \\
\text { camisas por } \\
\text { bandejas. }\end{array}$ & $\begin{array}{l}\text { Los tiradores de las } \\
\text { bandejas permiten } \\
\text { una mejor } \\
\text { manipulación de } \\
\text { éstas. }\end{array}$ & $\begin{array}{l}\text { El objeto al tener } \\
\text { bandejas que } \\
\text { comienzan desde el } \\
\text { suelo, dificultan el } \\
\text { accesos a éstas. }\end{array}$ \\
\hline & $\begin{array}{l}\text { Es compactable y se } \\
\text { puede colocar } \\
\text { horizontal y } \\
\text { verticalmente; Su } \\
\text { estructura permite } \\
\text { una fabricación } \\
\text { seriada. }\end{array}$ & $\begin{array}{l}\text { Módulos pequeños, } \\
\text { no permiten } \\
\text { almacenar una gran } \\
\text { cantidad de objetos. }\end{array}$ & $\begin{array}{l}\text { Es portable y se } \\
\text { puede utilizar según } \\
\text { las necesidades del } \\
\text { usuario. }\end{array}$ & $\begin{array}{l}\text { Al encontrarse en su } \\
\text { estado de máxima } \\
\text { extensión, se deben } \\
\text { retirar los objetos } \\
\text { contenidos para } \\
\text { poder compactarse. }\end{array}$ & $\begin{array}{l}\text { Su tamaño permite } \\
\text { la portabilidad. }\end{array}$ & $\begin{array}{l}\text { La manipulación del } \\
\text { mecanismo de } \\
\text { transformación } \\
\text { tiene libertades que } \\
\text { pueden confundir al } \\
\text { usuario a la hora del } \\
\text { despliegue de la } \\
\text { estructura. }\end{array}$ \\
\hline & $\begin{array}{l}\text { Estabilidad; en su } \\
\text { máxima extensión } \\
\text { permite almacenar } \\
\text { gran cantidad de } \\
\text { objetos; al tener una } \\
\text { forma cuadrada } \\
\text { ocupa al máximo el } \\
\text { espacio donde se } \\
\text { coloque; permite } \\
\text { fabricación seriada. }\end{array}$ & $\begin{array}{l}\text { Complejidad visual; } \\
\text { La estructura y } \\
\text { organización de los } \\
\text { módulos de } \\
\text { almacenamiento } \\
\text { obedece a una } \\
\text { estructura irregular } \\
\text { que no se condice } \\
\text { con un criterio de } \\
\text { clasificación por } \\
\text { tamaño. }\end{array}$ & $\begin{array}{lr}\text { Los módulos se } \\
\text { pueden configurar a } \\
\text { medida según se } \\
\text { necesite; } & \text { es } \\
\text { compactable. } & \end{array}$ & $\begin{array}{l}\text { Al compactarse en } \\
\text { su totalidad, se } \\
\text { requiere sacar todos } \\
\text { los elementos que } \\
\text { está conteniendo. }\end{array}$ & $\begin{array}{l}\text { Al generarse } \\
\text { espacios en toda su } \\
\text { extensión, vertical y } \\
\text { horizontal, permite } \\
\text { que personas de } \\
\text { cualquier estructura } \\
\text { lo puedan utilizar. }\end{array}$ & $\begin{array}{l}\text { Al no contar con un } \\
\text { manilla o ruedas, } \\
\text { resulta difícil de } \\
\text { extender }\end{array}$ \\
\hline
\end{tabular}

Fig. 1: Análisis del diseños de productos pre-existentes. Reproducida con autorización de Jara et al. (2013)

Ajuste de propuesta conceptual (opcional): Con base en el análisis de diseños pre-existentes y los productos existentes se ajusta la propuesta conceptual si llegara a ser necesario para que sea coherente y que constituya un aporte y novedad al contexto de la problemática.

Definición de modelos conceptuales: Considerando la propuesta conceptual y los atributos seleccionados de diseños pre-existentes se procede a definir los atributos y características del producto desagregadamente en forma, función y ergonomía. Tal como se puede apreciar en la figura 2. Estos modelos conceptuales buscan representar en una imagen isométrica los atributos y asociarlos a elementos de geometrías 
básicas; superficies, volúmenes, límites de contorno con el propósito de identificarlos y darles una ubicación relativa dentro del espacio del diseño.

FORM A

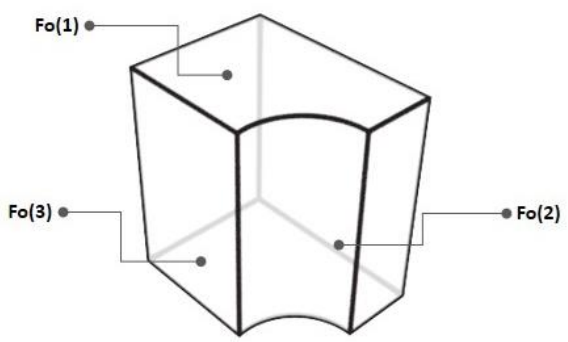

Fo(1)- Entre el objeto y el mesón debe respetarse una distancia mínima para la circulación de las trabajadoras.

Fo(2)- El ancho y altura del objeto deben estar limitados por la ventanilla de atención.

Fo(3)- Mientras la trabajadora esta sentada el objeto debe tener un rango de altura entre los $10 \mathrm{~cm}$ como mínimo y un máximo de $145 \mathrm{~cm}$, para un alcance inmediato.
FUNCIÓN

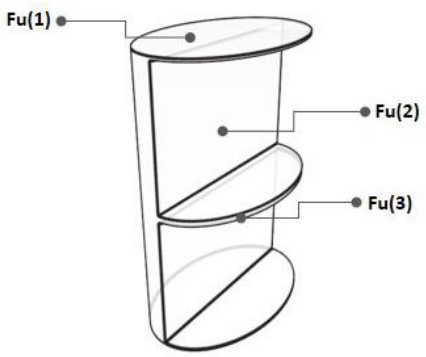

Fu(1)- El objeto debe contener aprovechando al máximo su extensión.

Fu(2)- El objeto debe tener una separación con el fin de diferenciar entre la ropa recibida y la ropa por entregar.

Fu(3)- Debe sujetar las bolsas de ropa de manera que no caigan durante la utilización.
E R G O N O MíA

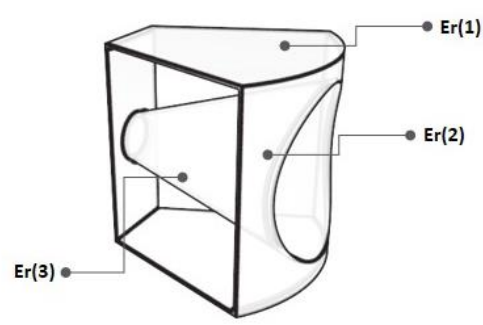

Er(1)- Debe tener una altura promedio acorde a las trabajadoras del local, para poder ser manipulable por éstas.

Er(2)-La zona de contención por la proyección del brazo del usuario sentado.

Er(3)-Debe proporcionar al usuario una inserción fluida de las bolsas.

Fig. 2: Modelos Conceptuales. Fuente: Reproducida con autorización de Jara et al. (2013)

Una vez que los atributos se hayan definido en cada uno de los subsistemas de forma, función y ergonomía se procederá a su representación geométrica de ellos utilizando para ello tres tipologías: volúmenes, superficies y límites de contorno.

\section{Integración del espacio del diseño}

El proceso se inicia valorando cada atributo de cada subsistema (forma, función y ergonomía) y las relaciones que entre éstos pueda existir. Junto con eso se esquematiza la integración en una representación análoga del espacio del diseño donde se van representando las geometrías principales de los atributos seleccionados. En este caso particular, como siguiente etapa los estudiantes tuvieron que construir una maqueta del espacio del diseño. La maqueta del espacio del diseño que se muestra en la figura 3 es en baja fidelidad y buscaba representar físicamente las geometrías representantes de los atributos fundamentales del producto: Volúmenes, Superficies y Límites de contornos de la forma, función y ergonomía. Este insumo tangible les facilitó la identificación de los atributos y de sus posibles consecuencias y efectos dentro de la propuesta de diseño.

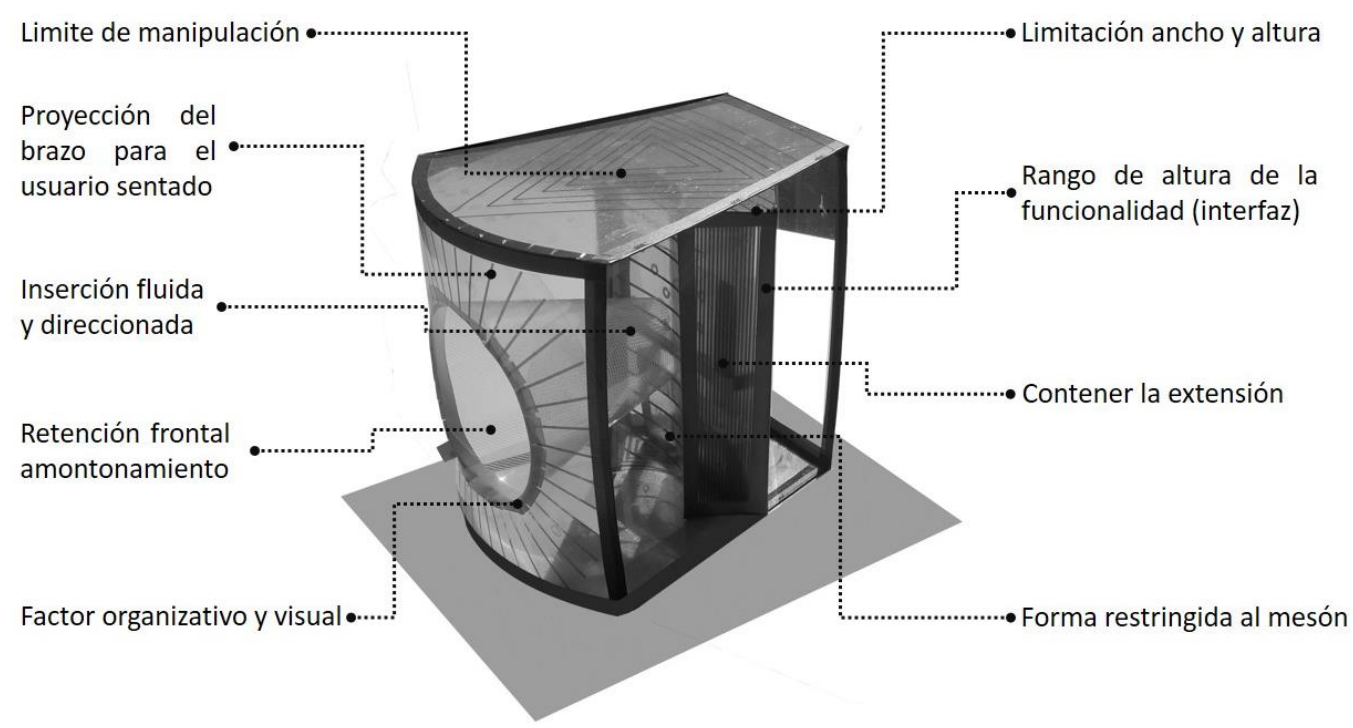

Fig. 3: Maqueta Espacio del Diseño. Fuente: Reproducida con autorización de Jara et al. (2013) 


\section{Desarrollo Formal y Propuesta Final}

Considerando la modelación del espacio del diseño así como los objetivos y requerimientos se procedió a la exploración de posibles formas y configuraciones del producto, como se puede observar en la figura 5. Dentro de este proceso se recogió la opinión y retroalimentación del usuario dentro de la elección, desarrollo y refinamiento de la propuesta de diseño.

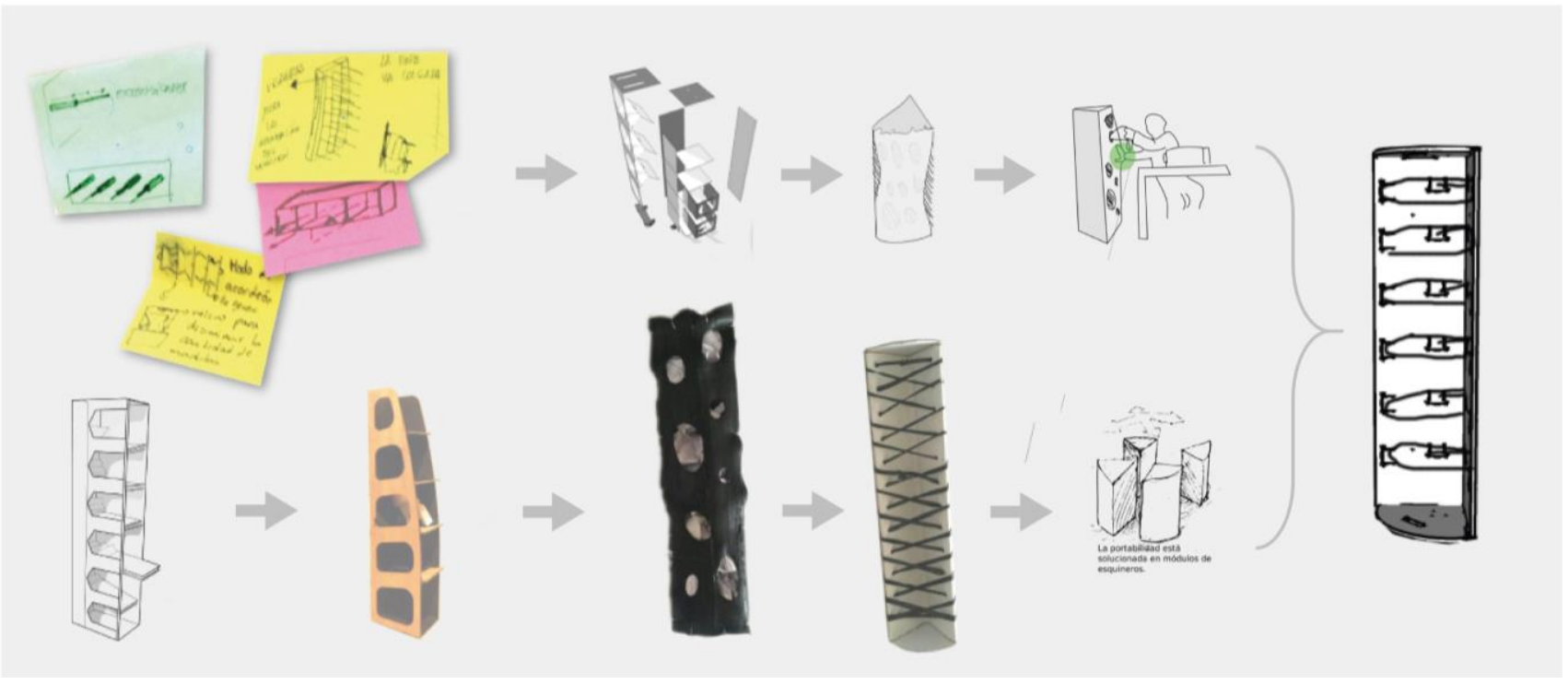

Fig. 4: Desarrollo formal y Prototipo de propuesta de diseño. Fuente: Reproducida con autorización de Jara et al. (2013)

\section{ESTUDIO}

Para caracterizar los resultados de la implementación del enfoque sistémico en la etapa de diseño conceptual, se realizó un estudio de alcance descriptivo con orientación exploratoria, mediante un análisis pre-experimental solo con post-test. Para recolectar los datos se aplicaron diferentes encuestas al final del taller, donde se evaluó la percepción de los estudiantes participantes respecto de la práctica del enfoque sistémico en el diseño y desarrollo de productos. La encuesta (mostrada en la Tabla 3 ) fue aplicada a 15 estudiantes de tercer año de la carrera de Diseño Industrial de la Universidad del Bío-Bío, la única universidad estatal de la ciudad de Concepción, Chile. Estos alumnos cursaban el taller [DCU] como parte de sus asignaturas obligatorias. Se obtuvo una muestra de quince participantes, ocho mujeres y siete hombres, de los 27 estudiantes que cursaron la asignatura. Fueron seleccionados mediante muestreo no probabilístico por accesibilidad y representan el 54,3\% del total de estudiantes.

Tabla 3: Cuestionario utilizado en la encuesta de percepción

\begin{tabular}{|c|c|c|c|c|c|}
\hline I. Metodología Sistémica & 1 & 2 & 3 & 4 & 5 \\
\hline 1. Aplicar la metodología sistémica me facilitó el proceso de análisis del producto por diseñar. & & & & & \\
\hline $\begin{array}{l}\text { 2. El análisis desagregado de las variables en forma, función y ergonomía me permitió identificar } \\
\text { aspectos claves del producto. }\end{array}$ & & & & & \\
\hline $\begin{array}{l}\text { 3. La metodología sistémica me permitió llevar un registro del proceso que permita fundamentar } \\
\text { las decisiones. }\end{array}$ & & & & & \\
\hline $\begin{array}{l}\text { 4. El modelo sistémico me ofreció una referencia teórica con los requerimientos y aspectos a } \\
\text { tener en consideración al momento de generar la propuesta de diseño formal }\end{array}$ & & & & & \\
\hline $\begin{array}{l}\text { 5. El análisis me permitió diferenciar los atributos o variables asociados a forma, función y } \\
\text { ergonomía }\end{array}$ & & & & & \\
\hline 6. La metodología sistémica me ayudó a comprender mejor el problema & & & & & \\
\hline II. Representación del espacio del diseño: & 1 & 2 & 3 & 4 & 5 \\
\hline $\begin{array}{l}\text { 1. La representación de los atributos en forma de geometrías me permitieron comprender la } \\
\text { relación entre los atributos identificados dentro del espacio. }\end{array}$ & & & & & \\
\hline $\begin{array}{l}\text { 2. Construir la maqueta tridimensional me permitió identificar la implicancia en el contexto real } \\
\text { de los atributos estudiados. }\end{array}$ & & & & & \\
\hline $\begin{array}{l}\text { 3. La maqueta del espacio de diseño fue un insumo que facilitó la comunicación y colaboración } \\
\text { con los miembros del equipo. }\end{array}$ & & & & & \\
\hline
\end{tabular}


El cuestionario aplicado en el proceso de encuesta que se puede apreciar en la tabla 3 incluía dos partes, una centrada en la percepción de la metodología sistémica y su utilidad en el proceso de diseño, y la segunda referida a la utilidad específica de dos herramientas empleadas durante la actividad: la representación de atributos en forma de geometrías y la construcción de una maqueta tridimensional. La encuesta presentaba nueve preguntas, seis de la primera parte y tres de la segunda parte del cuestionario, exhibiendo afirmaciones sobre el funcionamiento de la actividad. Para responder, los estudiantes debían emplear una escala Likert de cinco alternativas (1: Muy en desacuerdo; 2: En desacuerdo; 3: Indiferente; 4: De acuerdo; 5: Muy de acuerdo), según su percepción sobre lo vivenciado, ver Tabla 2. El cuestionario fue diseñado por los investigadores, revisado por los docentes de la asignatura y completado por los estudiantes que accedieron voluntariamente a hacerlo, de forma anónima y confidencial. Aunque sabían que el instrumento era parte de las actividades para retroalimentar el curso se les garantizó que sus respuestas no tendrían consecuencias para ellos.

\section{RESULTADOS}

En el siguiente apartado se exhiben los resultados obtenidos a partir de las opiniones y percepciones de los estudiantes con respecto a la contribución del uso de la metodología sistemática y la realización de la maqueta del espacio del diseño en la solución final de diseño. A continuación se detallan los resultados según los siguientes apartados:

\section{Evaluación global de la metodología sistémica:}

La Figura 5 indica que la aplicación de la metodología fue un recurso útil para los alumnos, porque les facilitó en primera instancia registrar bien el proceso, lo que contribuye claramente a la comprensión y capacidad de análisis para definir y jerarquizar las variables que participaban como atributos de valor del producto. Esta valoración realizada por los estudiantes corresponde a una comparativa con el ejercicio común que se realiza en aula, consistente en dialogar las variables y registrarlas verbalmente, pero que al no ser bien registradas ni ejecutadas en el espacio, generalmente se omiten o bien se jerarquizan erróneamente.

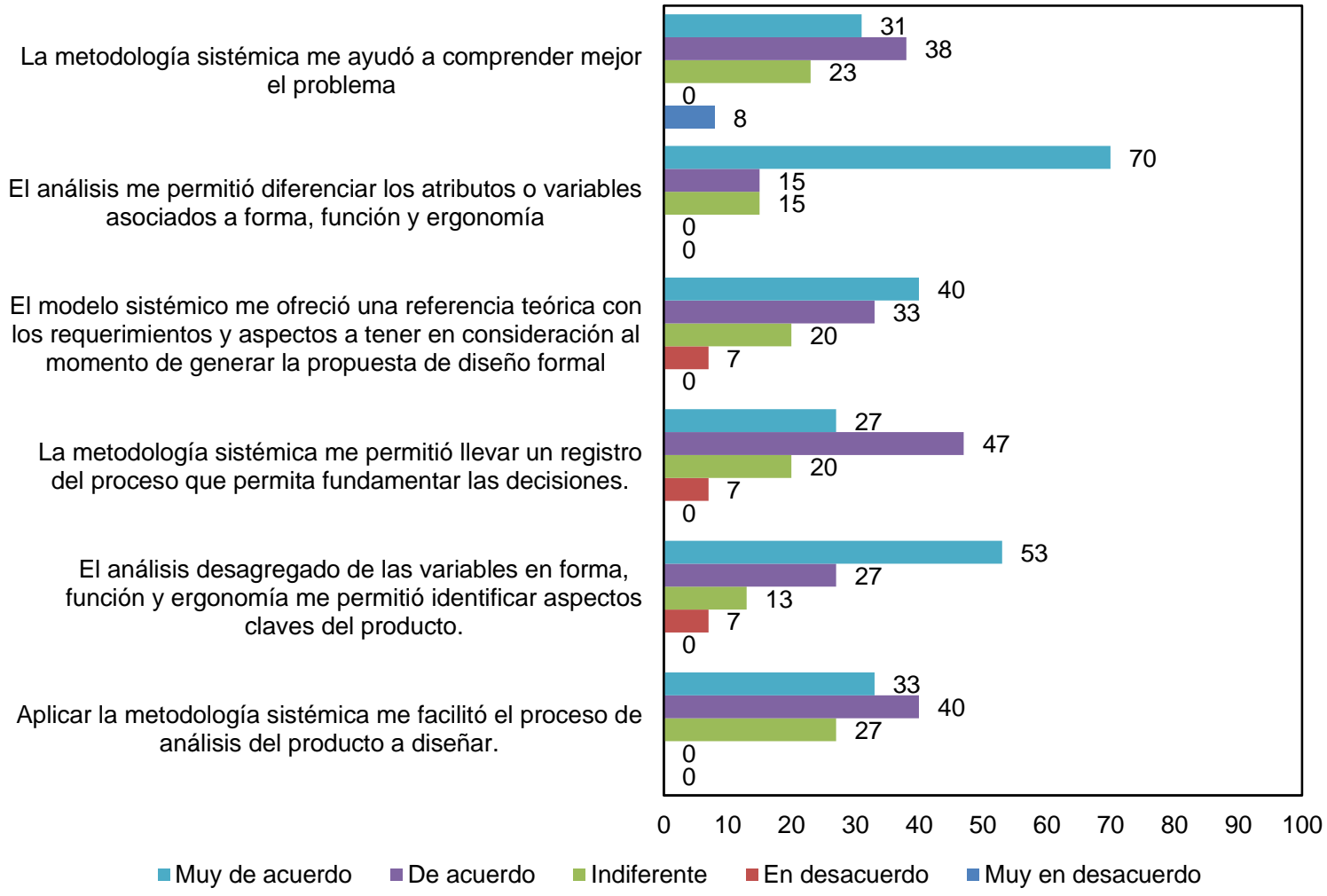

Fig. 5: Resultados de la percepción global (\%) de los estudiantes sobre el uso de la metodología sistémica

\section{Evaluación de herramientas específicas}

Los resultados expuestos en la Figura 6 muestran claramente cómo la elaboración de una maqueta tridimensional que expone atributos, facilita distinguirlos en su contexto. Sin embargo, existe discusión sobre si esta manera facilita la comunicación de ellos o la comprensión de estos atributos en el espacio de contexto. Esto fácilmente puede ser mejorado con la práctica y estudiado en detalle para distinguir aquellos factores que dificultan la apreciación y ventajes del modelo. 


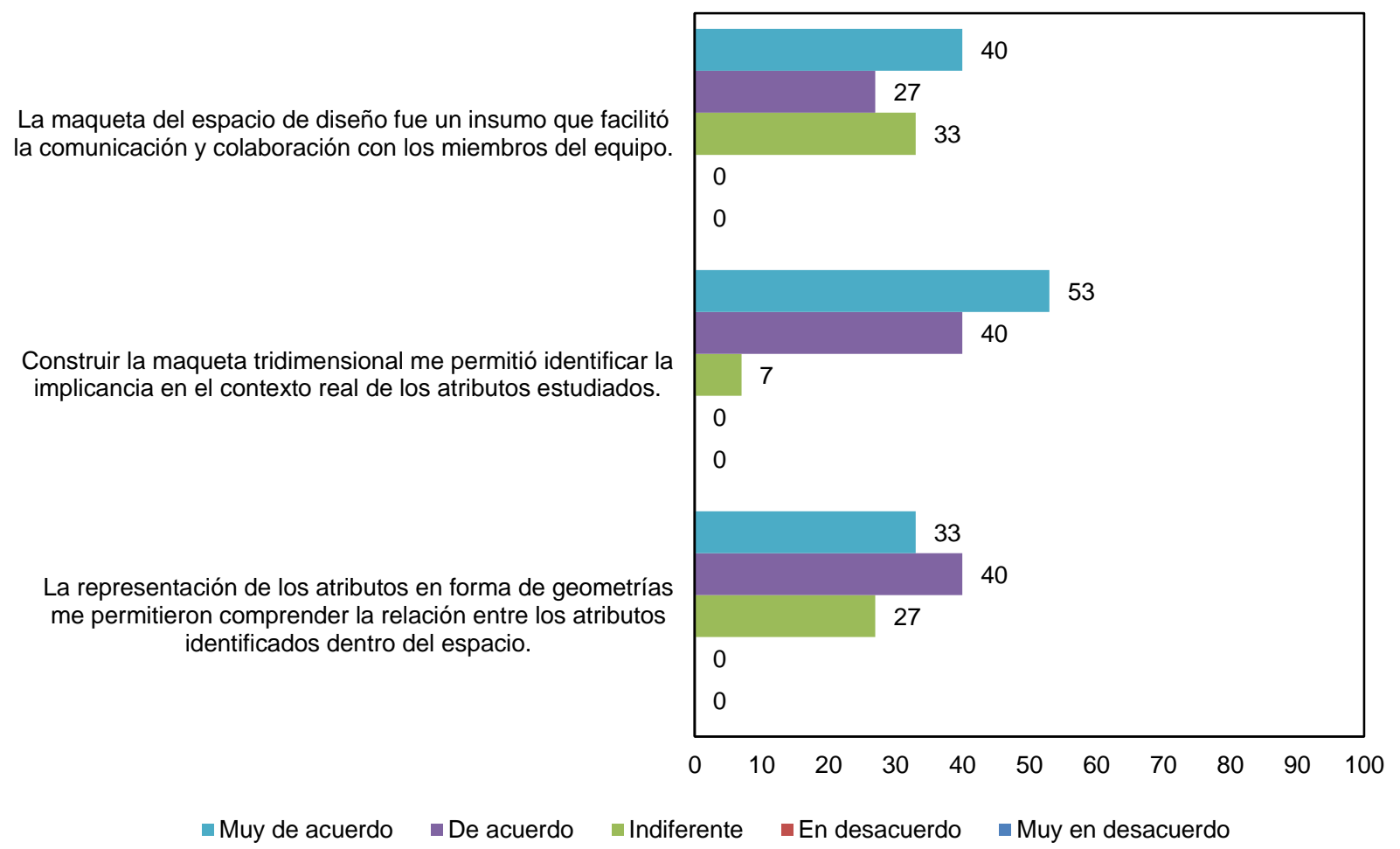

Fig.6: Evaluación de los estudiantes (\%) al uso de herramientas específicas durante la metodología sistémica

\section{DISCUSIÓN}

El objetivo del trabajo fue estudiar la percepción respecto de la aplicación del modelado sistémico y la representación del espacio del diseño en forma material a través de una maqueta en baja fidelidad. Dicha apuesta busca facilitar la identificación y gestión de los atributos del producto en la fase teórica/conceptual. La representación del espacio de diseño dentro del modelado sistémico permite relacionar dichas jerarquías y argumentos a su dimensión tangible, concentrando la atención del diseñador en aquellos conceptos relevantes, evidenciando de esta manera aquellas variables del diseño formal que serán coherentes con los conceptos y su respectiva argumentación lógica de valor sobre lo esperado del producto. Este último punto resulta ser uno de los más importantes de la propuesta metodológica, porque verbalizar un discurso en relación con su formalidad de manera coherente, implica una internalización clave y esencial que demuestra la transferencia de un conocimiento abstracto a una apuesta tangible y fundamentada. Esta transferencia será aplicable al mundo laboral, como una estrategia argumentativa que puede ser útil para el diseñador, ya sea para ofrecer su producto, como para resolver en distintos ámbitos, las soluciones más apropiadas a las diversas problemáticas y contextos. Estos beneficios fueron percibidos por los estudiantes en la encuesta aplicada, pues el evaluar globalmente la metodología, la mayoría tuvo una apreciación positiva de ésta obteniendose resultados similares a los de Hernandis y Briede (2009),principalmente, en cuanto a su utilidad para poder diferenciar forma, función y ergonomía, y cómo esta distinción permitió identificar aspectos clave del producto, lo que demuestra la utilidad de esta diferenciación en el proceso de diseño y de la metodología empleada para la aplicación de la misma.

Pese a esto, el aporte teórico y la utilidad del método sistémico para la comprensión del problema y el proceso de diseño son peor evaluadas por los estudiantes, lo que nuevamente puede enfrentarnos a como persiste la idea del diseño como un proceso intuitivo, lo que impide el uso y la valoración de herramientas teórico-procedimentales para dicho proceso, constatando lo ya evidenciado por Gudur (2016) relacionado con la dificultad de los estudiantes novicios para desarrollar el pensamiento abstracto.

Finalmente, en cuanto a las herramientas específicas, tanto las representaciones geométricas como la maqueta fueron muy bien evaluadas, aunque cerca de un tercio de los estudiantes se reportó indiferente al impacto de las primeras en comprender los atributos identificados dentro del espacio y a la utilidad de las maquetas para favorecer la comunicación y colaboración. Pese a esto, un $93 \%$ de los encuestados consideró que las maquetas permitían identificar la implicancia de los atributos en el contexto real, por lo que demuestran su utilidad para estos fines, llevando a cuestionarse de qué forma podría potenciarse también su utilidad comunicativa. Aunque la gran mayoría expuso su satisfacción al método, existe un grupo minoritario de estudiantes que mostraron desacuerdo con que la metodología ayudara a identificar aspectos claves del producto o aportara una referencia teórica, como tampoco sintieron que permitiera el registro del 
proceso y comprender mejor el problema. Si bien estos alumnos nunca representaban más de un $8 \%$ cabe cuestionarse si se debe a estudiantes que no lograron realizar una adecuada implementación de estas herramientas o, por el contrario, identificaron debilidades en la implementación de la misma. Por este motivo, para futuras instancias, se sugiere integrar herramientas cualitativas como entrevistas o grupos focales que permitan obtener una visión más experiencial y procesual de la implementación de estas herramientas.

\section{CONCLUSIONES}

La metodología sistémica permite el análisis detallado de tres variables fundamentales de un producto de diseño (forma, función y ergonomía), y los alumnos valoran que al separarlos se facilita la evaluación y jerarquización de sus condicionantes, relacionándolas, además, con el potencial comportamiento que el usuario espera del objeto en cuestión, esto aparece claramente reconocido por la mayoría de los estudiantes que participaron de la experiencia. También, en paralelo, tiene la ventaja de establecer criterios teóricos o experienciales sobre los que se fundamentan dichas jerarquizaciones, lo que racionaliza su puesta en valor. Siendo la maqueta una representación externa que puede facilitar la incorporación, identificación y posterior gestion de los atributos en un espacio volumétrico tridimensional concreto. Considerando que históricamente los docentes de los talleres de diseño siempre se enfrentan a la dificultad que tienen los estudiantes de convertir los valores conceptuales (que se pretenden abordar para mejorar un producto) a su conversión material, la realización de una maqueta como intermediaria puede facilitar la incorporación, identificación y posterior gestión de los atributos en un espacio volumétrico tridimensional concreto. El docente también se ve beneficiado porque el ejercicio de materialización (como proceso) y luego su construcción (como insumo físico de referencia), es claramente una ayuda al momento del evaluar el desarrollo formal en una dimensión concreta. Ayudando a todos los actores a diferenciar estas instancias claves dentro del proceso de diseño.

Desde luego esto es experimental y si bien el grupo de estudio es reducido en número, ya que considera a los estudiantes que aplicaron el enfoque durante el desarrollo del producto, permite identificar ciertas ventajas que habría que confirmarlas en un estudio más amplio para lograr representatividad, además sería interesante evaluar el método en talleres de distinto nivel y con diversas temáticas, pero es necesario destacar que cada vez que ha sido aplicado ha mostrado iguales beneficios didácticos. También este trabajo permite visualizar líneas de investigación respecto del efecto y/o contribución de las maquetas físicas, como también, a la reflexión en el proceso dialógico en la definición del espacio de diseño y su correlación con la propuesta de diseño conceptual.

\section{AGRADECIMIENTOS}

Los autores agradecen a la Vicerrectoría Académica de la Universidad del Bío-Bío a través del proyecto de Investigación e Innovación en Docencia UBB 2016-2017 código162004 /DdoC que pudo patrocinar este trabajo y al grupo de investigación en didáctica proyectual código Gl 160402/EF.

\section{REFERENCIAS}

Aguayo F. y V. Soltero, Metodología del diseño industrial: un enfoque desde la ingeniería concurrente. RaMa, p. 656. Madrid, España, ISBN: 9788478975327 (2002)

Asimow, M., Introduction to Design, Englewood Cliffs, N.J., Prentice-Hall (1962)

Babapour, M. Hiort Af Ornäs, V., Rexfelt, O. y U. Rahe, Media and representations in product design education, International Conference on Engineering and product Design Education, E\&PDE 4 \& September 2014, University of Twente, The Netherlands (2014)

Basu, S., Biswas, N., Naha Biswas S., Sarkar, S., A study on concurrent engineering-based design and product development, Inter. J. of Recent advances in Mechanical Engineering (IJMECH), 2(1), 15-20 (2013)

Briede, J.C., Cabello, M., Hernandis, B., Modelo de abocetado concurrente para el diseño conceptual de productos industriales, Dyna, 81(187), 199-208 (2014)

Briede, J.C., Cabello, M., Pacheco, B. y Cartes, J., Implicación de participantes en la fase de diseño conceptual de un producto. Caso de estudio del impacto de la metodología sistémica en una pyme chilena, Interciencia, 41(9), 622-628 (2016)

Briede, J.C., Cartes, J., Bustamante, A. y Pérez, M., "Shaping the individual designer: participatory design in emergency context," Proceedings of the $18^{\text {th }}$ International Conference on Engineering Design ICED11, Copenhagen, Denmark, 15-18 Aug., 8, 173-183 (2011) 
Coleman, K., Jansson, K., Kaiza P. y Reed, E., Homicides, Firearm Offences and Intimate violence, 2005/6: Supplementary Volume 1 to Crime in England and Wales 2005-6) (Home Office Statistical Bulletin 02/07, Office for National Statistics) (2007)

Correa, J., Pobreza, vulnerabilidad y calidad de vida en América Latina. Retos para la bioética, Acta Bioethica 2011, 17(1), 19-29, 20 (2011)

Corremans, J., Mulder-Nijkamp, M., Muses in Design: inspiration techniques for product form giving. 1르. Ed., Boom Editorial, Amsterdam, Netherlands, p. 320, 1 july (2016)

Gudur R.R., Challenges in teaching design thinking skills to novice design students. Proceedings of the $18^{\text {th }}$ International Conference on Engineering and Product Design Education (E\&PDE16), Design Education: Collaboration and Cross-Disciplinarity, Aalborg, Denmark, pp. 140-145, $8^{\text {th }}-9^{\text {th }}$ September (2016)

Hernandis B. y E., Iribarren, Diseño de Nuevos Productos, Una perspectiva sistémica. Cursos on-line. Formación Tutorizada a Distancia por Internet. Interf@d. Servicio de Publicaciones Universidad Politécnica de Valencia. $1^{\text {a }}$ Ed., Valencia, España (1999)

Hernandis, B., Desarrollo de una metodología sistemática para el diseño de productos industriales. Tesis Doctoral no publicada. Universidad Politécnica de Valencia, España (2003)

Hernandis, B., Opening ceremony, $6^{\text {th }}$ International Forum of Design as a Process. System \& Design. Beyond processes and thinking. Valencia, España (2016)

Hernandis, B. y J.C. Briede, An educational application for a product design and engineering systems using integrated conceptual models, Ingeniare. Revista chilena de ingeniería, 17(3), 432-442 (2009)

Hubka, V. y W.E. Eder, Design Science. Springer Verlag, Berlin, Heidelberg (1996)

Jara, D., S. Herrera y L. Palma, Esquinero modular expansible para el amontonamiento de ropa en espacios pequeños, Proyecto final Taller [DCU], Escuela de Diseño Industrial, Universidad del Bío-Bío (2013)

Jones, P., Systemic Design Principles for Complex Social Systems. Translational Systems Sciences book series (TSS, volumen 1). Social Systems and Design, 91-128 (2014)

Krippendorf, K., The semantic turn: a new foundation for design. Taylor \& Francis Group, 368 (2006)

Lim, Z., C. Anderson y S. McGrath, Professional Skills Development in a Resource- Poor Setting: The Case of Pharmacy in Malawi, International Journal of Educational Development, 32(5) 654-664 (2012)

Moyano, E., Castillo R. y J. Lizana, Trabajo informal: motivos, bienestar subjetivo, salud, y felicidad en vendedores ambulantes, Psicologia em Estudo, 13(4), 693-701 (2008)

Pahl, G. y W. Beitz, Engineering Design. A Systematic Approach. Ed. Springer Verlag (1996)

Rodríguez, J.C., El comercio ambulante como estrategia de emprendimiento comunitario (en línea: https://goo.gl/SC5gkJ, acceso: 30 enero 2017), Plataforma Urbana (2015)

Ryan, A., A Framework for Systemic Design, Form Akademisk, http://dx.doi.org/10.7577/formakademisk.787, https://goo.gl/4cBJgg, (4) 4, 1-14 (2014)

Silva, B., Opportunities and challenges in teaching Systemic Design The evoluation of the Open Systems master courses at Politecnico di Torino. IFDP`16 - Systems \& Design: Beyond Processes and Thinking. Universitat Politècnica de València, Spain, 57-66 (2016)

Systemic Design: Theory, Methods, and Practice (Translational Systems Sciences). Editors: Jones, Peter, Kijima, Kyoichi (Eds.) p. 94 (2018)

Tu, N., T. Zhang, Q. He, H. Zhang y Y. Li , User-Centered Design in New Product Development: A Case Study in Developing New Sports Earphone, International Conference on Computer and Management (CAMAN) 19-21 (2011)

Ulrich, K. y S. Eppinger, Product Design and Development. McGraw-Hill Higher Education; 5a Ed., p. 432 (2011)

Wilkinson, C.R., Applying user centred and participatory design approaches to commercial product development, Design Studies, 35(6), November, 614-631 (2014) 
